\title{
(2) OPEN ACCESS \\ Which treatment is most effective for patients with Achilles tendinopathy? A living systematic review with network meta-analysis of 29 randomised controlled trials
}

\author{
Arco C van der Vlist (1) , ${ }^{1}$ Marinus Winters (1) , ${ }^{2}$ Adam Weir, ${ }^{1,3}$ Clare L Ardern (ㄷ), ${ }^{4}$ \\ Nicky J Welton, ${ }^{5}$ Deborah M Caldwell, ${ }^{5}$ Jan A N Verhaar, ${ }^{1}$ Robert-Jan de Vos ${ }^{1}$
}

- Additional material is published online only. To view please visit the journal online (http://dx.doi.org/10.1136/ bjsports-2019-101872)

${ }^{1}$ Department of Orthopaedics and Sports Medicine, Erasmus University Rotterdam, Rotterdam, Zuid-Holland, The Netherlands

${ }^{2}$ Research Unit for General Practice in Aalborg, Department of Clinical Medicine, Aalborg University, Aalborg, Denmark

${ }^{3}$ Aspetar Sports Groin Pain Centre, Aspetar Qatar Orthopaedic and Sports Medicine Hospital, Doha, Ad Dawhah, Qatar ${ }^{4}$ Division of Physiotherapy, Karolinska Institute, Stockholm, Stockholm County, Sweden ${ }^{5}$ Population Health Sciences, Bristol Medical School, Bristol, UK

Correspondence to Arco C van der Vlist, Department of Orthopaedics and Sports Medicine, Erasmus University Rotterdam, 3000 CA Rotterdam, Zuid-Holland, The Netherlands;

a.vandervlist@erasmusmc.n

Accepted 4 May 2020 Published Online First 10 June 2020

\section{ABSTRACT}

Objective To provide a consistently updated overview of the comparative effectiveness of treatments for Achilles tendinopathy.

Design Living systematic review and network metaanalysis.

Data sources Multiple databases including grey literature sources were searched up to February 2019. Study eligibility criteria Randomised controlled trials examining the effectiveness of any treatment in patients with both insertional and/or midportion Achilles tendinopathy. We excluded trials with 10 or fewer participants per treatment arm or trials investigating tendon ruptures.

Data extraction and synthesis Reviewers independently extracted data and assessed the risk of bias. We used the Grading of Recommendations Assessment, Development and Evaluation to appraise the certainty of evidence.

Primary outcome measure The validated patientreported Victorian Institute of Sport Assessment-Achilles questionnaire.

Results 29 trials investigating 42 different treatments were included. 22 trials (76\%) were at high risk of bias and $7(24 \%)$ had some concerns. Most trials included patients with midportion tendinopathy (86\%). Any treatment class seemed superior to wait-and-see for midportion Achilles tendinopathy at 3 months (very low to low certainty of evidence). At 12 months, exercise therapy, exercise+injection therapy and exercise+night splint therapy were all comparable with injection therapy for midportion tendinopathy (very low to low certainty). No network meta-analysis could be performed for insertional Achilles tendinopathy.

Summary/conclusion In our living network metaanalysis no trials were at low risk of bias and there was large uncertainty in the comparative estimates. For midportion Achilles tendinopathy, wait-and-see is not recommended as all active treatments seemed superior at 3-month follow-up. There seems to be no clinically relevant difference in effectiveness between different active treatments at either 3-month or 12-month follow-up. As exercise therapy is easy to prescribe, can be of low cost and has few harms, clinicians could consider starting treatment with a calf-muscle exercise programme.

PROSPERO registration number CRD42018086467.

\section{INTRODUCTION}

The incidence of Achilles tendinopathy is $2-3$ per 1000 patients in general medicine practice, and its lifetime cumulative incidence can even increase to more than $50 \%$ in specific active populations (eg, runners). ${ }^{12}$ Achilles tendinopathy is an overload injury that is diagnosed clinically, and can affect the distal insertion or the midportion of the tendon. ${ }^{34}$ Managing tendinopathy is challenging. Patients can expect their symptoms to improve between 3 and 12 months after commencing treatment, but not beyond 12 months. ${ }^{5}$ Chronic symptoms persist in approximately a quarter of patients 10 years after treatment, and tendinopathy impairs both quality of life and physical activity. ${ }^{56}$

Wait-and-see, exercise therapy, injections, shockwave therapy, orthosis, medication and surgery are the main treatment options offered to patients with Achilles tendinopathy. ${ }^{7-14}$ Most patients receive multiple treatments over time, thereby impacting on healthcare consumption. ${ }^{15}$ Trials and conventional meta-analysis do not directly assess the relative effectiveness of all available treatments, challenging patients and clinicians when they make treatment decisions. Network meta-analysis (NMA) allows the simultaneous comparison of the effectiveness of all treatments and can rank treatments from most to least effective. ${ }^{1617}$ As no comprehensive NMA of all treatment options for Achilles tendinopathy exists, our aim was to evaluate the comparative effectiveness of all available treatments for Achilles tendinopathy in a regularly updated ('living') systematic review using NMA.

\section{METHODS}

\section{Protocol and registration}

Our living systematic review with NMA was prospectively registered on PROSPERO (International Prospective Register of Systematic Reviews) and published in an open repository. ${ }^{18}$ We followed the Preferred Reporting Items for Systematic Reviews and Meta-Analyses (PRISMA) guideline, the Meta-Analysis of Observational Studies in Epidemiology guideline, and the PRISMA-NMA extension for reporting NMA. ${ }^{19-21}$

\section{Administration and update of the living} systematic review

This systematic review with NMA is part of the Dutch multidisciplinary guideline for Achilles 
tendinopathy. As we (1) plan to update the search and review process every 5 years as part of the guideline revision process, and (2) perform annual screening to identify new data that may alter the conclusions and recommendations, ${ }^{18}$ we defined this as a 'living' systematic review with NMA. A living systematic review's main advantage is that it assumes that new knowledge will appear and allow improvements in clinical decision-making. As we already have a structured protocol and database for this systematic review, we will be able to answer future research questions quickly and promote faster translation of new scientific evidence into clinical practice.

\section{Patient involvement}

To determine clinically relevant outcomes, we performed a pilot round of focus interviews with consecutive patients suffering from chronic midportion Achilles tendinopathy $(n=9)$ who were participating in a randomised clinical trial (NCT02996409), and administered a survey to patients identified through the Dutch national patient federation $(n=97) .{ }^{17}$ The results are presented in online supplementary web appendix 1 . We also considered recently defined core domains for tendinopathies from an international consensus involving patients and healthcare providers. ${ }^{22}$ Based on these data, we decided to evaluate clinical outcome with the validated and disease-specific Victorian Institute of Sport Assessment-Achilles (VISA-A) score as the primary outcome. $^{1823}$

\section{Outcome measures}

The VISA-A score quantifies pain and activity level and can range from 0 to 100; a score of 100 indicates no pain with full activity level, while a score of 0 indicates severely limited activity levels and severe levels of pain. The minimal important difference (MID) for the VISA-A score is 15 points. ${ }^{24}$ Return to sports activities was the secondary outcome. We assessed outcomes at 3, 6 and 12 months.

\section{Eligibility criteria}

Trials were eligible if they investigated the effectiveness of any treatment in adults ( $\geq 18$ years) with Achilles tendinopathy, using the outcome measures VISA-A questionnaire and/or return to sports activities. Populations with midportion tendinopathy, insertional tendinopathy or a combination of both were included. Achilles tendinopathy must have been diagnosed based on clinical findings (eg, local pain reproduced on clinical examination). ${ }^{25}$ Imaging to confirm the diagnosis was not an inclusion criterion. Trials including athletes and/or inactive patients were eligible. There were no language restrictions.

Any treatment, control treatment, placebo, wait-and-see or no treatment group studied in a trial was eligible for inclusion. We predefined a number of treatment classes, based on the assumption that some treatments have a similar effect due to a comparable working mechanism. ${ }^{18}$ Table 1 shows the treatments that are subdivided into treatment classes.

We excluded trials with (1) 10 or fewer participants per study arm, (2) an inadequate control group (eg, the use of the contralateral Achilles tendon), (3) a population with full-thickness ruptures of the Achilles tendon, and (4) animal or in vitro studies.

\section{Literature search strategy and information source}

We developed a sensitive search strategy for multiple databases with the assistance of a medical librarian (online supplementary web appendix 2). The following databases were searched for published and unpublished trials up to 21 February 2019: Embase, MEDLINE Ovid, Web of Science, Cochrane CENTRAL, CINAHL EBSCOhost, SPORTDiscus EBSCOhost, AMED EBSCOhost, WHO ICTRP, ClinicalTrials.gov, WorldCat.org, OpenGrey and Google Scholar. We used the validated Cochrane search filter 'Embase search strategy for finding randomised clinical trials in Embase', and modified this for all conventional databases. ${ }^{26}$ We screened the reference lists of all included publications for potentially eligible trials.

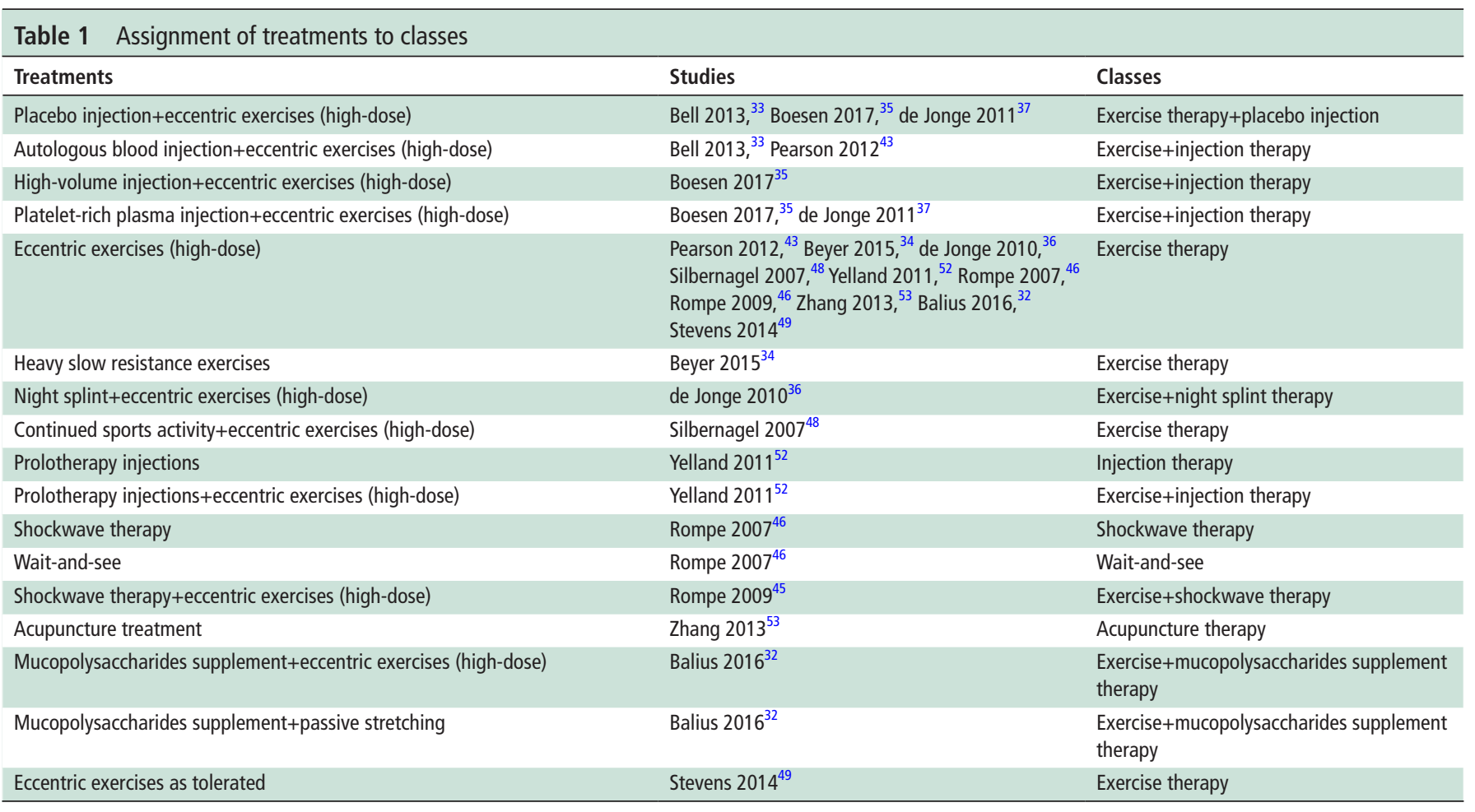


Study selection and data extraction

Titles and abstracts were screened by two independent reviewers (ACV and RJ-V) after duplicate removal. Disagreements were resolved by consensus. Two reviewers independently applied eligibility criteria to the full text reports. Disagreements were resolved by a third reviewer (AW). In case of unpublished records, authors were contacted for data availability. We uploaded all trials to Covidence (Veritas Health Innovation, Melbourne, Australia).

Data were extracted independently by two reviewers (ACV plus one of AW, CA or RJ-V) using standardised extraction forms adapted from the Cochrane Collaboration. ${ }^{26}$ Disagreements were resolved by consensus, or a fifth reviewer (MW) in case of persistent disagreement. We extracted publication and trial details, population characteristics, eligibility criteria, treatment details, relevant outcome information, details of analysis, and study authors' key conclusions. If there were multiple time points available within a study, and these were equally close to the time point being synthesised across trials, we extracted the outcome of the latest follow-up for analysis. ${ }^{18}$

\section{Risk of bias assessment}

We used the Risk of Bias 2 tool to assess risk of bias for each trial outcome. ${ }^{27}$ We assessed risk of bias on the basis of 'assignment to intervention' for all five domains: (1) randomisation process, (2) deviations from intended interventions, (3) missing outcome data, (4) outcome measurement and (5) selection of the reported result. An overall risk of bias judgement was made for each outcome and each time point as either 'low risk', 'some concerns' or 'high risk' of bias. ${ }^{27} 28$

The assessment was performed independently by two reviewers (ACV plus one of MW, CLA, AW or RJ-V). The reviewers did not perform risk of bias assessment or data extraction for publications in which they were involved as author. Disagreements were resolved via consensus or by a third reviewer (MW or AW) if necessary.

\section{Data synthesis and statistical methods}

We constructed network plots using Stata V.15 to visualise all head-to-head comparisons. Eccentric exercises were labelled as high-dose (daily) or low-dose (less than once a day). We planned a time course analysis for the VISA-A score; however, insufficient data precluded the analysis. ${ }^{18}$ Instead, we modelled networks for the primary and secondary outcomes at 3 months, 6 months and 12 months, where possible. Four reviewers (ACV, $\mathrm{MW}, \mathrm{AW}$ and R-JV) labelled the treatments and assigned them to categories (classes) (see table 1). We assessed the assumption of exchangeability required for NMA before commencing the analyses. We appraised clinical homogeneity by tabulating study and population characteristics and inspecting them for differences in effect modifiers.

Treatment-level and class-level models were fitted in a Bayesian framework using Markov chain Monte Carlo simulations in WinBUGS (V.1.4; Medical Research Council, UK, and Imperial College of Science, Technology and Medicine, University of Cambridge, UK). ${ }^{17} 29$ Continuous outcomes are presented as mean difference, with their $95 \%$ credible intervals. We reported the mean, median and $95 \%$ credible intervals for the ranking of each treatment or class to estimate the likelihood of individual treatments being superior to other treatments, and interpreted the primary outcome results in light of the MID.

For both treatment-level and class-level models, we fitted fixed and random effects models, and compared model fit using the deviance information criterion and posterior mean residual deviance. ${ }^{17}$ Lower deviances depict a better model fit. For the class-level models we attempted to fit a hierarchical model where treatment effects were assumed to be similar within class, but due to insufficient data we were only able to fit a fixed class effect model where all treatments were assumed to have the same effect within class. ${ }^{17}$

We assessed statistical heterogeneity by inspecting the betweentrial SD and comparing the fit of the fixed and random effects models. ${ }^{17}$ We planned to explore sources of statistical heterogeneity if there were 10 or more trials available per comparison. In the presence of evidence from direct and indirect comparisons, it is important to assess whether the direct and indirect evidence is consistent. ${ }^{30}$ We assessed the consistency assumption for each network by comparing model fit between the NMA model and an unrelated mean effects model that relaxes the consistency assumption. ${ }^{30}$ We planned to assess small study bias using comparison-adjusted funnel plots if 10 or more trials were available for one comparison.

\section{Certainty of evidence}

We used the Grading of Recommendations Assessment, Development and Evaluation (GRADE) for NMAs to appraise the certainty of evidence. ${ }^{31}$ Evidence from each comparison was appraised for direct and indirect comparisons, and for the combined evidence, where applicable. Evidence could be of 'high certainty', 'moderate certainty', 'low certainty' or 'very low certainty'. All ratings started at the level of 'high certainty'. Two authors (ACV and MW) independently rated the evidence down on the basis of risk of bias, inconsistency, indirectness, imprecision and publication bias. Inconsistency in GRADE involves heterogeneity across trials and is not related to inconsistency in the network. We rated the overall body of evidence in the network to indicate the strength of the NMA recommendations.

\section{RESULTS}

We identified 5,154 potentially relevant publications, of which 170 articles were screened in the full-text analysis. Twenty-nine trials $(\mathrm{n}=1,640$ patients) met our eligibility criteria and were included (figure 1). ${ }^{32-60}$ Online supplementary web appendix 3 lists the 14 unpublished trials and the 3 trials awaiting classification.

\section{Characteristics of the included trials}

Forty-two treatments were investigated in 29 trials. Sixty-five treatment arms were included in the trials, and 40 of these included exercise therapies. There were 180 treatment comparisons in the NMAs. The majority of trials (86\%) investigated midportion Achilles tendinopathy, one investigated insertional Achilles tendinopathy and three trials did not specify the location of Achilles tendinopathy. Twenty-five trials evaluated clinical outcome using the VISA-A score and six trials reported return to sports activities. Sample sizes ranged from 12 to 117 per treatment arm (median 20 patients, IQR 16-27). Follow-up duration ranged from 1 to 52 weeks (median 27 weeks, IQR 12-52).

The baseline characteristics of patients and all trial characteristics are shown in online supplementary web appendices 4 and 5. Study populations reflect clinical practice with equal sex distribution, participants in their 40s and slightly overweight. Three-quarters of the included participants were active individuals. Participants typically had symptoms for nearly 2 years prior to treatment; approximately $20 \%$ had bilateral symptoms. 


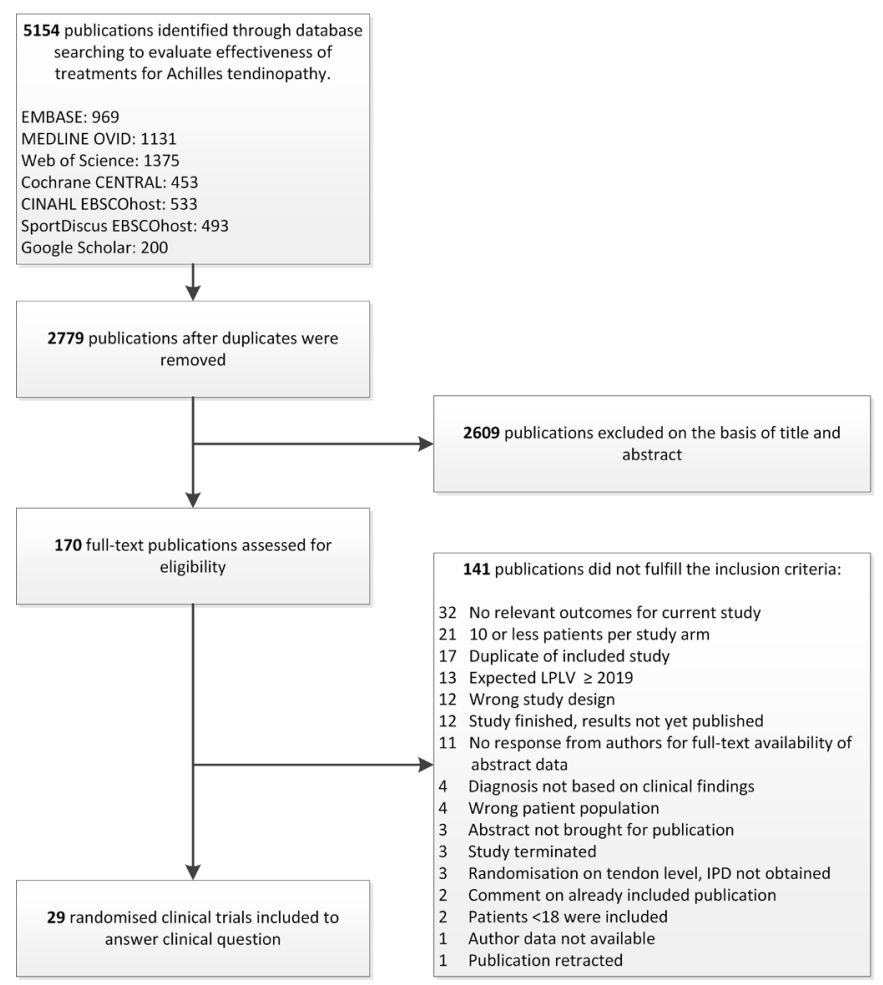

Figure 1 PRISMA flow diagram of the study selection process. IPD, individual patient data; LPLV, last patient last visit; PRISMA, preferred reporting items for systematic reviews and meta-analyses.

The severity of symptoms measured with the VISA-A score was usually between 40 and 60 points.

\section{Risk of bias and certainty of evidence}

Twenty-two trials (76\%) were at high risk of bias (online supplementary web appendix 6). We had some concerns about bias in seven trials (24\%). No trials were at low risk of bias. In studies that used both the VISA-A score and return to sport as outcome measures, there was no difference in risk of bias between the outcomes. In $48 \%$ of the trials, outcome measurement was a source of bias. All other sources of bias were also commonly judged as high risk: the randomisation procedure (21\%), deviations from the intended intervention (28\%), missing outcome data $(28 \%)$ and selection of reported results $(24 \%)$.

Certainty of evidence for all comparisons was low to very low, except for autologous blood+eccentric exercise therapy versus placebo injection+eccentric exercise therapy, for which there was moderate certainty of evidence (online supplementary web appendix 7). The main reasons to rate down the certainty of evidence were study limitations $(n=180,100 \%)$ and imprecision $(\mathrm{n}=158,88 \%)$. We did not rate down for inconsistency, indirectness or publication bias. Only two treatment comparisons were studied in multiple (ie, 2) trials (ie, platelet-rich plasma injection +eccentric exercise therapy vs placebo injection + eccentric exercise therapy, and laser +eccentric exercise therapy vs placebo laser+eccentric exercise therapy). Where this was the case, estimates and credible intervals had substantial overlap. Populations, treatments and outcome measures followed those used in clinical practice; hence, there was no indication of indirectness in the evidence. We did not assess publication bias because there were fewer than 10 trials available for each of the comparisons.
A)

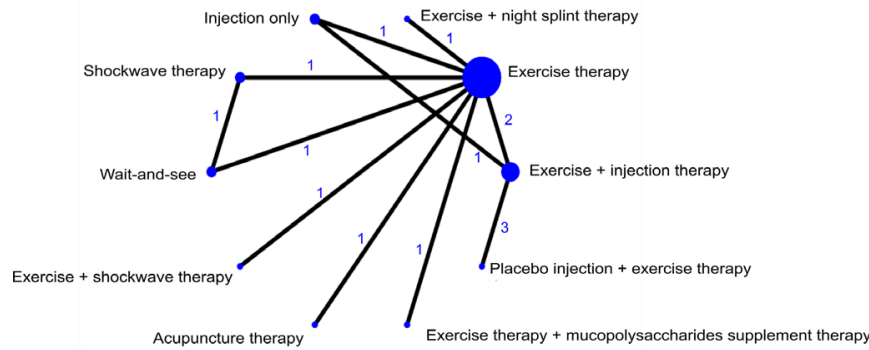

B)

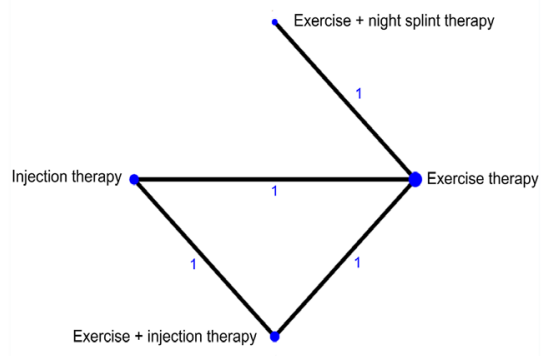

Figure 2 Network plots for treatment classes on the VISA-A score at 3 and 12 months in patients with midportion Achilles tendinopathy. The size of the dots is proportional to the number of participants who received the treatment, respectively. Blue numbers indicate the number of trials the classes were compared in. Note that intraclass comparisons are not included in the plot (eg, eccentric exercises vs heavy slow resistance exercises); all treatment comparisons can be found in online supplementary appendix 9. (A) VISA-A score at 3 months. (B) VISA-A score at 12 months. VISA-A, Victorian Institute of Sport AssessmentAchilles.

\section{Network meta-analyses}

Figure 2A,B shows direct treatment class comparisons in the field of midportion Achilles tendinopathy for VISA-A score. Model fit statistics are reported in online supplementary web appendix 8. Ten classes were included in the network analyses. None of the networks included evidence from both direct and indirect comparisons, so the consistency assumption for NMA could not be checked. Figure 3 shows the comparative treatment class effects on the VISA-A score at 3 months (figure $3 \mathrm{~A}$ ) and 12 months (figure 3B). Figures 4 and 5 show the treatment class rankings for 3 and 12 months. No class analyses could be performed for the VISA-A score at 6 months and return to sports activities at 6 months (only time point in NMA) due to insufficient evidence (online supplementary web appendix 8). Treatment effect NMAs for VISA-A and return to sports activities are presented in online supplementary web appendix 9 . No NMA could be performed for insertional Achilles tendinopathy. Studies that could not be included in the NMA are presented in online supplementary web appendix 10 .

\section{Comparative treatment class effectiveness on the primary} outcome (VISA-A score)

VISA-A score at 3 months

Seventeen treatments, studied in 13 randomised controlled trials (RCTs), were assigned to 10 classes. Any treatment seemed superior to wait-and-see: exercise+placebo injection therapy (mean difference 19 points, 95\% CrI -3 to 34 


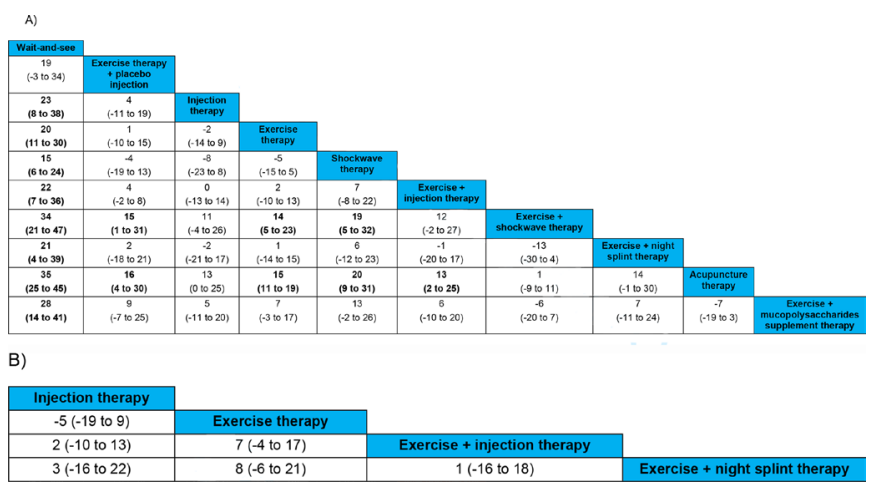

Figure 3 Comparative treatment class effects expressed with a mean difference for the VISA-A score at 3 months (A) and at 12 months (B) in patients with midportion Achilles tendinopathy. Mean differences on the VISA-A score with their 95\% credible intervals from the network metaanalysis. For any cell, a negative mean difference favours the upper-left treatment, and a positive mean difference favours the lower-right treatment. Comparative treatment class effect differences are shown in bold. VISA-A, Victorian Institute of Sport Assessment-Achilles.

points), injection therapy (23 points, 8 to 38 points), exercise therapy (20 points, 11 to 30 points), shockwave therapy (15 points, 6 to 24 points), exercise+injection therapy (22 points, 7 to 36 points), exercise + shockwave therapy (34 points, 21 to 47 points), exercise + night splint therapy ( 21 points, 4 to 39 points), acupuncture therapy (35 points, 25 to 45 points) and mucopolysaccharides supplement+exercise therapy (28 points, 14 to 41 points) (figure $3 \mathrm{~A}$ ).

Acupuncture therapy seemed superior to placebo injection therapy (16 points, 4 to 30 points), injection therapy (13 points, 0 to 25 points), exercise therapy (15 points, 11 to 19 points), shockwave therapy (20 points, 9 to 31 points), exercise+injection therapy (13 points, 2 to 25 points), exercise + night splint therapy (14 points, -1 to 30 points) and mucopolysaccharides supplement+exercise therapy ( 7 points, -3 to 19 points), but not to exercise + shockwave therapy (1 point, -9 to 11 points).

Exercise+shockwave therapy seemed superior to placebo injection therapy (15 points, 1 to 31 points), injection therapy (11 points, -4 to 26 points), exercise therapy (14 points, 5 to 23 points), shockwave therapy alone (19 points, 5 to 32 points), exercise+injection therapy (12 points, -2 to 27 points) and exercise + night splint therapy ( 13 points, -4 to 30 points), but not to acupuncture therapy ( -1 point, -11 to 9 points) and

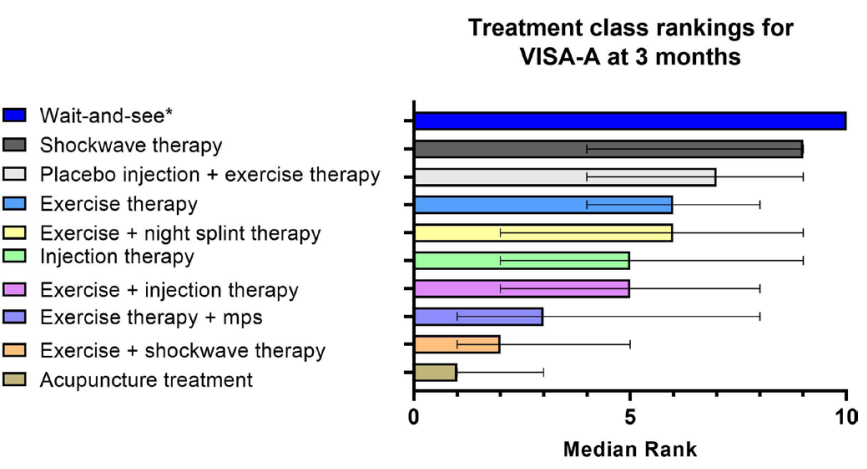

Figure 4 Treatment class rankings from the network meta-analysis for the VISA-A score at 3 months in patients with midportion Achilles tendinopathy. The asterix indicates that the $95 \%$ credible interval was rank 10 to 10 for wait-and-see therapy. MPS, mucopolysaccharides supplement; VISA-A, Victorian Institute of Sport Assessment-Achilles.
Treatment rankings for VISA-A at 12 months

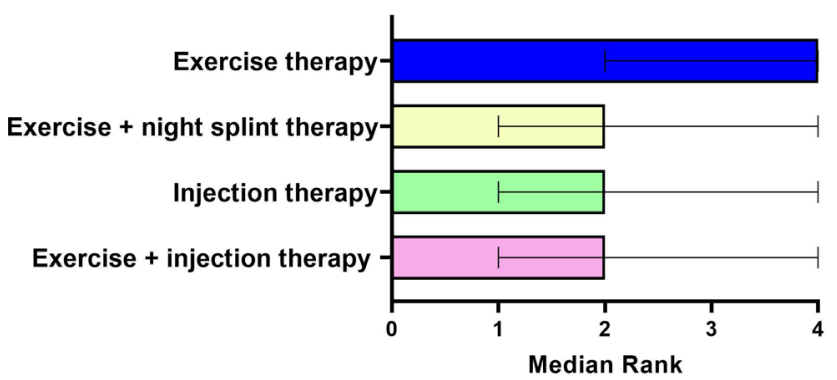

Figure 5 Treatment class rankings from the network meta-analysis for the VISA-A score at 12 months in patients with midportion Achilles tendinopathy. VISA-A, Victorian Institute of Sport Assessment-Achilles.

mucopolysaccharides supplement+exercise therapy (6 points, -7 to 20 points).

\section{VISA-A score at 12 months}

At 12 months, six treatments, studied in four RCTs, were assigned to four treatment classes and compared in a network model. Exercise therapy ( -5 points, -19 to 9 points), exercise+injection therapy ( 2 points, -10 to 13 points) and exercise + night splint therapy ( 3 points, -16 to 22 points) were all comparable with injection therapy (figure $3 \mathrm{~B}$ ).

\section{Treatment class rankings}

According to the NMA rankings, acupuncture therapy seemed the best treatment at 3 months (mean ranking 1.56, median ranking 1, median's 95\% CrI 1 to 3 ), and exercise+shockwave therapy seemed second best (mean ranking 1.98, median ranking 2, median's 95\% CrI 1 to 5). At 12 months, injection therapy (mean ranking 2.47, median ranking 2, median's 95\% CrI 1 to 4), exercise therapy (mean ranking 3.51, median ranking 4 , median's 95\% CrI 2 to 4), exercise +injection therapy (mean ranking 2.03, median ranking 2, median's 95\% CrI 1 to 4) and exercise + night splint therapy (mean ranking 1.99, median ranking 2 , median's 95\% CrI 1 to 4 ) had similar rankings (figures 4 and 5).

Comparative treatment class effectiveness on the secondary outcome (return to sports activities)

No class analyses could be performed for return to sports activities at 6 months (only time point for NMA) due to insufficient evidence. The results of the treatment-level analyses for return to sports activities at 6 months are presented in online supplementary web appendix 9 .

\section{DISCUSSION}

In this living systematic review and NMA, none of the trials were at low risk of bias, and all evaluated treatments had large uncertainty in the estimates. From a myriad of treatments for midportion Achilles tendinopathy, active treatment classes seem to have clinically meaningful benefits (mean difference exceeded VISA-A MID of 15 points) at 3 months compared with wait-and-see. ${ }^{24}$ For two classes (acupuncture therapy, and shockwave therapy combined with exercise therapy), the credible intervals exceeded the MID of 15 points. However, these results were based on two small trials (64 and 68 included patients, respectively) at high risk of bias. There were no estimates for effectiveness of wait-and-see at 12 months. The effectiveness of most active treatments in the long term is uncertain. At 12 months, there 
was no difference between exercise therapy, injection therapies and combined therapies.

\section{Clinical implications}

Based on the findings in this study, we advise against recommending wait-and-see therapy as a treatment strategy. Active treatments had overlapping comparative effects, leaving uncertainty about which treatment is best for Achilles tendinopathy. Shared decision-making plays an important role in managing Achilles tendinopathy. Safety profile, treatment availability and costs should be considered in this clinical decision-making process. Calf-muscle exercise therapy is easy to prescribe because it is easy to instruct, can be cheap, is available everywhere and has a low risk of harm. ${ }^{14} 61$

The preinjury activity level could play an important role in the response to treatments such as exercise therapy as this will possibly influence the load that can be applied to the tendon. There is currently no tool available to distinguish sedentary from athletic patients, and people define this differently. When a patient is considered to be sedentary, the VISA-A score could be less sensitive to changes as $40 \%$ of the score can be obtained from sporting activities. ${ }^{23}$ Therefore, a novel modified version of the existing VISA-A questionnaire is currently being developed to evaluate treatment response in sedentary individuals. ${ }^{62}$ In two trials, lower baseline VISA-A score was associated with a greater increase in VISA-A score. ${ }^{3748}$ This is most likely explained by regression to the mean (outliers will tend to move towards the mean score). Furthermore, physical activity level was not a prognostic factor for change in VISA-A score in the trials that attempted to measure physical activity. ${ }^{33}$ More research is needed to determine whether athletic and sedentary patients should be considered separate patient groups.

\section{Strengths and weaknesses in relation to other studies}

The uncertainty around the effectiveness for specific treatments has previously been described in Achilles tendinopathy. Most recent meta-analyses on treatment effectiveness for Achilles tendinopathy did not find superiority of a single treatment. ${ }^{8} 1063-65$ All other systematic reviews included randomised clinical trials or quasi-randomised controlled clinical trials and made strong to weak recommendations in favour of exercise therapy, ${ }^{8}$ platelet-rich plasma ${ }^{65}$ or against platelet-rich plasma ${ }^{10}$ or splinting ${ }^{8}$ single therapies. Our NMA results conflict with a recent systematic review with head-to-head meta-analysis where prolotherapy and sclerotherapy were superior to other treatments. ${ }^{13}$ Differences in study selection criteria (case series were also included vs only randomised clinical trials in our NMA) and outcome measure selection (eg, general Visual Analogue Scale vs a validated patient-reported outcome measure in our NMA) may explain the discrepancy.

\section{Unanswered questions and future research}

As uncertainty around treatment effectiveness remains, future trials should be adequately powered and designed. Universally agreed diagnostic criteria for both insertional and midportion Achilles tendinopathy are needed to prevent heterogeneity in included participants. More trials investigating treatment effects for insertional Achilles tendinopathy are warranted. New trials should include exercise therapy as a stand-alone comparator with long-term ( $\geq 12$ months) follow-up. This would facilitate assessment of the comparative effectiveness of new treatments in the network. Based on our findings it is not ethical to perform new studies using wait-and-see as a treatment arm.
The role of patient education has never been explored, but should be further assessed. Trialists must include measures from the core outcome set for tendinopathy (patient overall rating, participation, pain on activity/loading, disability, function, physical function capacity, quality of life, psychology and pain over a specified timeframe). ${ }^{22}$ We identified eight ongoing trials on the effectiveness of multiple treatments for Achilles tendinopathy. We aim to perform horizon scanning annually to screen for recently published trials. In case of relevant trials, we will update the analyses and publish the results when there are important changes that affect clinical care. We plan to update this 'living' systematic review and NMA at least every 5 years as part of the Dutch national guideline revision process.

\section{Strengths and limitations}

We compared treatments in an NMA despite some of these treatments having never been compared head-to-head in trials. Our living NMA will be updated at least every 5 years, which allows for a contemporary evidence synthesis for clinical practice. ${ }^{6667}$ The protocol was registered prospectively and patients were involved in selecting outcomes most relevant to them in the design phase.

The small sample sizes and bias in the included trials limit our conclusions. ${ }^{68}$ Many of the investigated treatments were not connected to the network, which hampered assessing the comparative effectiveness of all available treatments. Sources of heterogeneity could not be investigated as there were less than the required 10 trials per comparison. None of the comparisons in the networks had both direct and indirect evidence and so we could not check the consistency. ${ }^{17}$

Treatment outcomes may be different on the basis of some patient characteristics and previous Achilles tendinopathy treatment. For example, a sedentary population may respond to exercise treatment differently compared with an athletic population. Other effect modifiers may be unilateral/bilateral symptoms, duration of symptoms and type/number of previous treatments. However, to the best of our knowledge, there is presently no evidence supporting any of these factors as treatment effect modifiers. The limited amount of study-level data precluded investigating effect modification. This may be possible in the future using pooled individual patient data.

We excluded many trials because they did not use commonly accepted and validated outcomes in the field of Achilles tendinopathy. ${ }^{69}$ It is unclear whether our NMA results apply to all patients with Achilles tendinopathy because we only included one trial with patients with insertional Achilles tendinopathy. We planned a time course analysis for the VISA-A score, as outcomes within the first year are of equal importance. Due to a lack of data, we were not able to perform this analysis and, in accordance with our protocol, we modelled outcomes for 3 months, 6 months and 12 months instead.

We planned threshold analysis as a quantitative means to assess the robustness of NMA recommendations to potential limitations in the evidence. We were unable to use this approach because of substantial overlap in credible intervals from the NMA. Due to overlap in the intervals, no recommendations could be made, which is a fundamental prerequisite to performing a valid threshold analysis. To comply with our protocol, we report threshold results in online supplementary web appendix 11 , but chose to use GRADE to interpret the evidence. We were not able to evaluate small study bias due to too low number of trials. We found three completed trials in trial registers; two are under review, and the publication status of one trial is unknown. 
Therefore, it seems unlikely that, in an era of prospective trial registrations, significant publication bias is present in the field of Achilles tendinopathy.

\section{CONCLUSION}

Our living NMA of 29 RCTs on Achilles tendinopathy included 42 different treatments. No trials were at low risk of bias, most had only short follow-up, and there was large uncertainty in the comparative estimates. For midportion Achilles tendinopathy, active treatments seem superior to wait-and-see at 3-month follow-up. There was no evidence of a clinically relevant difference in effectiveness between different active treatments at 3-month and 12-month follow-up. Calf-muscle exercise therapy is easy to prescribe in practice, is widely available, and is regarded as safe and cheap. Consequently, clinicians should consider starting this as initial treatment.

\section{What is already known}

Achilles tendinopathy is a common, persisting condition where one in four patients still has symptoms after 10 years.

- Achilles tendinopathy can be disabling and impacts on quality of life.

- Many patients receive multiple treatments, which represents high healthcare consumption.

- The comparative effectiveness of treatments for Achilles tendinopathy is currently unknown.

\section{What are the new findings}

- In our living network meta-analysis of randomised controlled trials of patients with Achilles tendinopathy, wait-and-see is not recommended as all active treatments were found to be superior to it.

- Given the large uncertainty in comparative estimates and risk of bias in the studies, no specific treatment can be recommended over another.

Twitter Arco C van der Vlist @ArcoVlist, Marinus Winters @marinuswinters, Clare L Ardern@clare_ardern and Robert-Jan de Vos@ri_devos

Acknowledgements We would like to extend our gratitude to WM Bramer, research librarian at Erasmus MC, for his help with developing a sensitive search strategy. The Dutch Patient Federation assisted in sending, receiving and extracting surveys that were completed online by patients who suffered self-reported Achilles tendinopathy. We are grateful for their help and input in the design of the survey, thereby improving knowledge on important and relevant outcome domains for patients with Achilles tendinopathy. We thank Andreas Serner (Aspetar Orthopaedic and Sports Medicine Hospital) for screening the full texts of non-English publications. We thank Michael Skovdal Rathleff, Henrik Riel and Mads Hilligsøe (Centre for General Practice at Aalborg University, Aalborg, Denmark) for their help in conducting data extraction tables for publication.

Contributors MW, AW and R-JV came up with the study idea. ACV, MW, AW, CLA, NJW, DMC, JANV and R-JV were involved in designing the study. ACV and $\mathrm{R}$-JV performed the literature search and screened trials for inclusion. ACV, MW, AW CLA and R-JV performed the risk of bias assessment and data extraction. MW, NJW and DMC designed the statistical analysis plan and analysed the data. ACV, MW, AW and R-JV interpreted the results of the data analyses. ACV, MW, AW and R-JV drafted the manuscript. R-JV is the study guarantor. All authors reviewed the final manuscript and agreed to be accountable for all aspects of the work and approved the final manuscript for submission. The corresponding author attests that all listed authors meet the authorship criteria and that no others meeting the criteria have been omitted.

Funding This research received a grant from the Dutch Association of Medical Specialists to develop a clinical guideline for the treatment of patients with Achilles tendinopathy. The Dutch Patient Federation is involved in this guideline development and assisted in sending out patient surveys.
Competing interests NJW led a research project in collaboration with Pfize (project ended 31 December 2018). Pfizer part-funded a junior researcher. The projects were purely methodological, using historical data on pharmacological treatments for pain relief.

\section{Patient consent for publication Not required.}

Provenance and peer review Not commissioned; externally peer reviewed.

Open access This is an open access article distributed in accordance with the Creative Commons Attribution Non Commercial (CC BY-NC 4.0) license, which permits others to distribute, remix, adapt, build upon this work non-commercially, and license their derivative works on different terms, provided the original work is properly cited, appropriate credit is given, any changes made indicated, and the use is non-commercial. See: http://creativecommons.org/licenses/by-nc/4.0/.

\section{ORCID iDs}

Arco C van der Vlist http://orcid.org/0000-0003-4238-3540

Marinus Winters http://orcid.org/0000-0001-5742-7441

Clare L Ardern http://orcid.org/0000-0001-8102-3631

\section{REFERENCES}

1 de Jonge $S$, van den Berg $C$, de Vos RJ, et al. Incidence of midportion achilles tendinopathy in the general population. Br J Sports Med 2011;45:1026-8.

2 Kujala UM, Sarna S, Kaprio J. Cumulative incidence of achilles tendon rupture and tendinopathy in male former elite athletes. Clin J Sport Med 2005;15:133-5.

3 Cook JL, Purdam CR. Is tendon pathology a continuum? A pathology model to explain the clinical presentation of load-induced tendinopathy. Br J Sports Med 2009:43:409-16.

4 de Vos RJ, D'Hooghe P, de Leeuw P, et al. Chapter 19: Achilles tendinopathy. In: The ankle in football. 1 edn. Paris: Springer-Verlag Paris, 2014: p213-33.

5 Lagas IF, Tol JL, Weir A, et al. One in four patients with midportion achilles tendinopathy has persisting symptoms after 10 years: a prospective cohort study. Am J Sports Med 2019

6 Ceravolo ML, Gaida JE, Keegan RJ. Quality-of-life in achilles tendinopathy: an exploratory study. Clin J Sport Med 2018. doi:10.1097/JSM.0000000000000636. [Epub ahead of print: 13 Aug 2018].

7 Challoumas D, Kirwan PD, Borysov D, et al. Topical glyceryl trinitrate for the treatment of tendinopathies: a systematic review. Br J Sports Med 2019;53:251-62.

8 Wilson F, Walshe M, O'Dwyer T, et al. Exercise, orthoses and splinting for treating achilles tendinopathy: a systematic review with meta-analysis. Br J Sports Med 2018:52:1564-74.

9 Murphy M, Travers M, Gibson W, et al. Rate of improvement of pain and function in mid-portion achilles tendinopathy with loading protocols: a systematic review and longitudinal meta-analysis. Sports Med 2018:48:1875-91.

10 Zhang Y-J, Xu S-Z, Gu P-C, et al. Is platelet-rich plasma injection effective for chronic achilles tendinopathy? A meta-analysis. Clin Orthop Relat Res 2018;476:1633-41.

11 Chen X, Jones IA, Park C, et al. The efficacy of platelet-rich plasma on tendon and ligament healing: a systematic review and meta-analysis with bias assessment. Am J Sports Med 2018;46:2020-32

12 Korakakis $\mathrm{V}$, Whiteley $\mathrm{R}$, Tzavara A, et al. The effectiveness of extracorporeal shockwave therapy in common lower limb conditions: a systematic review including quantification of patient-rated pain reduction. Br J Sports Med 2018:52:387-407.

13 Morath 0, Kubosch EJ, Taeymans J, et al. The effect of sclerotherapy and prolotherapy on chronic painful achilles tendinopathy-a systematic review including meta-analysis. Scand J Med Sci Sports 2018:28:4-15.

14 Murphy MC, Travers MJ, Chivers P, et al. Efficacy of heavy eccentric calf training for treating mid-portion achilles tendinopathy: a systematic review and meta-analysis. $\mathrm{Br}$ J Sports Med 2019;53:1070-7

15 van der Plas A, de Jonge S, de Vos RJ, et al. A 5-year follow-up study of Alfredson's heel-drop exercise programme in chronic midportion Achilles tendinopathy. Br J Sports Med 2012:46:214-8.

16 Ioannidis J. Next-generation systematic reviews: prospective meta-analysis, individuallevel data, networks and umbrella reviews. Br J Sports Med 2017;51:1456-8.

17 Dias S, Ades AE, Welton NJ, et al. Network meta-analysis for comparative effectiveness research. Wiley: Hoboken NJ, 2018.

18 Winters M, Holden S, Vicenzino B, et al. Which treatment is most effective for patients with patellofemoral pain? A protocol for a living systematic review including network meta-analysis. BMJ Open 2018:8:e022920.

19 Moher D, Shamseer L, Clarke M, et al. Preferred reporting items for systematic review and meta-analysis protocols (PRISMA-P) 2015 statement. Syst Rev 2015;4:1.

20 Hutton B, Salanti G, Caldwell DM, et al. The PRISMA extension statement for reporting of systematic reviews incorporating network meta-analyses of health care interventions: checklist and explanations. Ann Intern Med 2015;162:777-84.

21 Stroup DF, Berlin JA, Morton SC, et al. Meta-analysis of observational studies in epidemiology: a proposal for reporting. meta-analysis of observational studies in epidemiology (MOOSE) group. JAMA 2000;283:2008-12.

22 Vicenzino $\mathrm{B}$, de Vos R-J, Alfredson $\mathrm{H}$, et al. Icon 2019-International scientific tendinopathy symposium consensus: there are nine core health-related domains for 
tendinopathy (core domains): Delphi study of healthcare professionals and patients. Br J Sports Med 2020;54:444-51.

23 Robinson JM, Cook JL, Purdam C, et al. The VISA-A questionnaire: a valid and reliable index of the clinical severity of achilles tendinopathy. Br J Sports Med 2001;35:335-41.

24 Lagas IF, van der Vlist $A C$, van Oosterom RF, et al. The minimal clinically important difference and patient acceptable symptom state of the VISA-A score for achilles tendinopathy. Clin Orthop Relat Res 2019.

25 Scott A, Squier K, Alfredson H, et al. ICON 2019: international scientific tendinopathy symposium consensus: clinical terminology. Br J Sports Med 2020;54:260-2.

26 Higgins JPT, Green S. Data Collections Forms for intervention reviews. In: Cochrane handbook for systematic reviews of interventions. The Cochrane Collaboration, 2011. http://training.cochrane.org/resource/data-collection-forms-intervention-reviews

27 Sterne JAC, Savović J, Page MJ, et al. RoB 2: a revised tool for assessing risk of bias in randomised trials. BMJ 2019:366:14898.

28 Higgins JPT, Sterne JAC, Savović J, et al. A revised tool for assessing risk of bias in randomized trials. Cochrane Database Syst Rev 2016:10:29-31.

29 Dias S, Sutton AJ, Ades AE, et al. Evidence synthesis for decision making 2: a generalized linear modeling framework for pairwise and network meta-analysis of randomized controlled trials. Med Decis Making 2013;33:607-17.

30 Dias S, Welton NJ, Sutton AJ, et al. Evidence synthesis for decision making 4: inconsistency in networks of evidence based on randomized controlled trials. Med Decis Making 2013;33:641-56.

31 Guyatt GH, Oxman AD, Vist GE, et al. GRADE: an emerging consensus on rating quality of evidence and strength of recommendations. BMJ 2008;336:924-6.

32 Balius R, Álvarez G, Baró F, et al. A 3-arm randomized trial for achilles tendinopathy: eccentric training, eccentric training plus a dietary supplement containing mucopolysaccharides, or passive stretching plus a dietary supplement containing mucopolysaccharides. Curr Ther Res Clin Exp 2016;78:1-7.

33 Bell KJ, Fulcher ML, Rowlands DS, et al. Impact of autologous blood injections in treatment of mid-portion achilles tendinopathy: double blind randomised controlled trial. BMJ 2013;346:f2310.

34 Beyer R, Kongsgaard M, Hougs Kjær B, et al. Heavy slow resistance versus eccentric training as treatment for achilles tendinopathy: a randomized controlled trial. Am J Sports Med 2015:43:1704-11.

35 Boesen AP, Hansen R, Boesen MI, et al. Effect of high-volume injection, plateletrich plasma, and sham treatment in chronic midportion achilles tendinopathy: a randomized double-blinded prospective study. Am J Sports Med 2017;45:2034-43.

36 de Jonge S, de Vos RJ, Van Schie HTM, et al. One-year follow-up of a randomised controlled trial on added splinting to eccentric exercises in chronic midportion achilles tendinopathy. Br J Sports Med 2010;44:673-7.

37 de Jonge S, de Vos RJ, Weir A, et al. One-year follow-up of platelet-rich plasma treatment in chronic achilles tendinopathy: a double-blind randomized placebocontrolled trial. Am J Sports Med 2011;39:1623-9.

38 Herrington L, McCulloch R. The role of eccentric training in the management of achilles tendinopathy: a pilot study. Physical Therapy in Sport 2007:8:191-6.

39 Hutchison AM, Pallister I, Evans RM, et al. Intense pulsed light treatment of chronic mid-body achilles tendinopathy: a double blind randomised placebo-controlled trial. Bone Joint J 2013;95-B:504-9.

40 Krogh TP, Ellingsen T, Christensen $\mathrm{R}$, et al. Ultrasound-Guided injection therapy of achilles tendinopathy with platelet-rich plasma or saline: a randomized, blinded, placebo-controlled trial. Am J Sports Med 2016;44:1990-7.

41 Lynen N, De Vroey T, Spiegel I, et al. Comparison of peritendinous hyaluronan injections versus extracorporeal shock wave therapy in the treatment of painful achilles' tendinopathy: a randomized clinical efficacy and safety study. Arch Phys Med Rehabil 2017;98:64-71.

42 Munteanu SE, Scott LA, Bonanno DR, et al. Effectiveness of customised foot orthoses for achilles tendinopathy: a randomised controlled trial. Br J Sports Med 2015;49:989-94.

43 Pearson J, Rowlands D, Highet R. Autologous blood injection to treat achilles tendinopathy? A randomized controlled trial. J Sport Rehabil 2012;21:218-24.

44 Rompe JD, Furia J, Maffulli N. Eccentric loading compared with shock wave treatment for chronic insertional achilles tendinopathy: a randomized, controlled trial. J Bone Joint Surg Am 2008;90:52-61.

45 Rompe JD, Furia J, Maffulli N. Eccentric loading versus eccentric loading plus shockwave treatment for midportion achilles tendinopathy: a randomized controlled trial. Am J Sports Med 2009;37:463-70
46 Rompe JD, Nafe B, Furia JP, et al. Eccentric loading, shock-wave treatment, or a wait-and-see policy for tendinopathy of the main body of tendo achillis: a randomized controlled trial. Am J Sports Med 2007;35:374-83

47 Roos EM, Engström M, Lagerquist $A$, et al. Clinical improvement after 6 weeks of eccentric exercise in patients with mid-portion achilles tendinopathy -- a randomized trial with 1-year follow-up. Scand J Med Sci Sports 2004;14:286-95.

48 Silbernagel KG, Thomeé R, Eriksson BI, et al. Continued sports activity, using a pain-monitoring model, during rehabilitation in patients with achilles tendinopathy: a randomized controlled study. Am J Sports Med 2007;35:897-906.

49 Stevens M, Tan C-W. Effectiveness of the alfredson protocol compared with a lower repetition-volume protocol for midportion achilles tendinopathy: a randomized controlled trial. J Orthop Sports Phys Ther 2014;44:59-67.

50 Tumilty S, Mani R, Baxter GD. Photobiomodulation and eccentric exercise for achilles tendinopathy: a randomized controlled trial. Lasers Med Sci 2016;31:127-35.

51 Tumilty S, McDonough S, Hurley DA, et al. Clinical effectiveness of low-level laser therapy as an adjunct to eccentric exercise for the treatment of achilles' tendinopathy: a randomized controlled trial. Arch Phys Med Rehabil 2012;93:733-9.

52 Yelland MJ, Sweeting KR, Lyftogt JA, et al. Prolotherapy injections and eccentric loading exercises for painful achilles tendinosis: a randomised trial. $\mathrm{Br}$ J Sports Med 2011;45:421-8.

53 Zhang B-meng, Zhong L-wei, Xu S-wei, et al. Acupuncture for chronic achilles tendnopathy: a randomized controlled study. Chin J Integr Med 2013;19:900-4.

54 Auclair J, Georges M, Grapton X, et al. A double-blind controlled multicenter study of percutaneous niflumic acid gel and placebo in the treatment of achilles heel tendinitis. Eur J Rheumatol Inflamm 1991;46:782-8.

55 Njawaya MM, Moses B, Martens D, et al. Ultrasound quidance does not improve the results of shock wave for plantar fasciitis or calcific achilles tendinopathy: a randomized control trial. Clin J Sport Med 2018;28:21-7.

56 Usuelli FG, Grassi M, Maccario C, et al. Intratendinous adipose-derived stromal vascular fraction (SVF) injection provides a safe, efficacious treatment for achilles tendinopathy: results of a randomized controlled clinical trial at a 6-month follow-up. Knee Surg Sports Traumatol Arthrosc 2018;26:2000-10.

57 Ebbesen BH, Mølgaard CM, Olesen JL, et al. No beneficial effect of polidocanol treatment in achilles tendinopathy: a randomised controlled trial. Knee Surg Sports Traumatol Arthrosc 2018:26:2038-44

58 Heinemeier KM, Øhlenschlæger TF, Mikkelsen UR, et al. Effects of antiinflammatory (NSAID) treatment on human tendinopathic tissue. J App/ Physiol 2017;123:1397-405.

59 Morrison RJM, Brock TM, Reed MR, et al. Radiofrequency microdebridement versus surgical decompression for achilles tendinosis: a randomized controlled trial. J Foot Ankle Surg 2017;56:708-12.

60 Silbernagel KG, Thomeé $\mathrm{R}$, Thomeé $\mathrm{P}$, et al. Eccentric overload training for patients with chronic achilles tendon pain--a randomised controlled study with reliability testing of the evaluation methods. Scand J Med Sci Sports 2001:11:197-206.

61 Martin RL, Chimenti R, Cuddeford T, et al. Achilles pain, stiffness, and muscle power deficits: midportion achilles tendinopathy revision 2018. J Orthop Sports Phys Ther 2018;48:A1-38.

62 Raju J, Norris R, Gaida JE. 015: development and validation of the VISA-A(sedentary) questionnaire: a modified version of the VISA-A for nonathletic patients with achilles tendinopathy, 2016

63 Kearney RS, Parsons N, Metcalfe D, et al. Injection therapies for achilles tendinopathy. Cochrane Database Syst Rev 2015:CD010960

64 Lin M-T, Chiang C-F, Wu C-H, et al. Meta-analysis comparing autologous bloodderived products (including platelet-rich plasma) injection versus placebo in patients with Achilles tendinopathy. Arthroscopy 2018:34:1966-75.

65 Liu C-J, Yu K-L, Bai J-B, et al. Platelet-rich plasma injection for the treatment of chronic achilles tendinopathy: a meta-analysis. Medicine 2019:98:e15278.

66 Vandvik PO, Brignardello-Petersen R, Guyatt GH. Living cumulative network metaanalysis to reduce waste in research: a paradigmatic shift for systematic reviews? BMC Med 2016;14:59.

67 Elliott JH, Turner T, Clavisi O, et al. Living systematic reviews: an emerging opportunity to narrow the evidence-practice gap. PLoS Med 2014;11:e1001603.

68 Weir A, Rabia S, Ardern C. Trusting systematic reviews and meta-analyses: all that glitters is not gold! Br J Sports Med 2016;50:1100-1.

69 Macdermid JC, Silbernagel KG. Outcome evaluation in tendinopathy: foundations of assessment and a summary of selected measures. J Orthop Sports Phys Ther 2015;45:950-64 